\title{
PROTEUS SYNDROME: REPORT OF A CASE WITH VASCULAR MALFORMATION SUCCESSFULLY TREATED WITH ENDOVENOUS RADIOFREQUENCY ABLATION
}

\author{
Patchsariya Prongchantuk*, Anucha Panoi**, Boonchai Boonyawat***, Pajaree Thitthiwong*** \\ *Department of Internal Medicine, Phramongkutklao College of Medicine, Bangkok, Thailand \\ ** Department of Surgery, Phramongkutklao College of Medicine, Bangkok, Thailand \\ ***Department of Pediatrics, Phramongkutklao College of Medicine, Bangkok, Thailand
}

\begin{abstract}
We reported a case of a 10 year-old Thai boy diagnosed as Proteus syndrome, presented with progressive enlarging of extremities with asymmetric disproportionate overgrowth and distorting of the feet and toes. Skin examination showed marked thickening of the soles without characteristic cerebriform appearance, and welldefined purplish plaques with capillary-microcystic lymphatic malformation on the right hip and thigh. MRI/MRA of extremities showed disproportionate overgrowth with combined lymphatic-venous malformation at the right buttock and right lower extremity. He was treated with endovenous radiofrequency ablation (EV-RFA) by using VNUS $^{\mathbb{1}}$ Radiofrequency Generator Model RFG2 (VNUS Medical Technologies Inc., San Jose, California, USA and foam sclerotherapy after ablation as an adjunctive treatment for the venous malformation of the right leg with favorable outcomes.
\end{abstract}

Keywords : Proteus syndrome, Cerebriform connective tissue nevus, Endovenous radiofrequency ablation

J Southeast Asian Med Res 2017;1:25-28.

http://www.jseamed.org

\section{Correspondence to:}

Thitthiwong P, Department of Pediatrics, Phramongkutklao College of Medicine, Bangkok, Thailand E-mail : pajareepmk@gmail.com 


\section{Introduction}

Proteus syndrome is characterized by variable manifestations, including vascular malformations, cerebriform connective tissue nevus, epidermal nevi, lipomas or dysregulated adipose tissue, dermal hypoplasia, asymmetric disproportionate overgrowth of body parts, facial phenotype and hyperpigmentation. Cerebriform connective tissue nevus is the pathognomonic sign of Proteus syndrome. Proteus syndrome is caused by an activating somatic mutation of the AKTl gene (c. 49G > A or p. Glu17Lys). The clinical variability emphasizes the mosaic basis for this syndrome. Treatment of Proteus syndrome is challenging.

\section{Results}

We reported a case of a 10 year-old Thai boy presented with progressive enlarging and distorting of extremities with the diagnosis of Proteus syndrome. He had enlarged and thickened feet with hemihypertrophy of the right buttock and right leg. Asymmetric disproportionate overgrowth and distorting of the toes of both feet were observed with slightly difficulty walking. He can do activities of daily living independently, however, with potential functional impairment. His parents reported that the patient had normal developmental milestones and intelligence. He was born to a healthy mother with term, vaginal delivery with 2,800 grams birthweight. There was neither family history of a similar skin lesion nor known genetic disorders in the family. There was no consanguineous marriage in the family, shown in the pedigree as Fig 1 .

His facial appearance was also dysmorphic, including dolichocephaly, long face and downslanting palpebral fissures. Dermatologic examination showed marked thickening of both soles without the characteristic cerebriform appearance and well-defined purplish plaques with clinically capillary-microcystic lymphatic malformation on the right hip and right thigh. The large protruding abdominal mass with superficial vein dilatation was also observed.

The MRI of abdomen found fatty overgrowth along left lateral abdominal wall with splenomegaly. The clinical signs in this patient were shown in Fig 2,3,4 and 5.

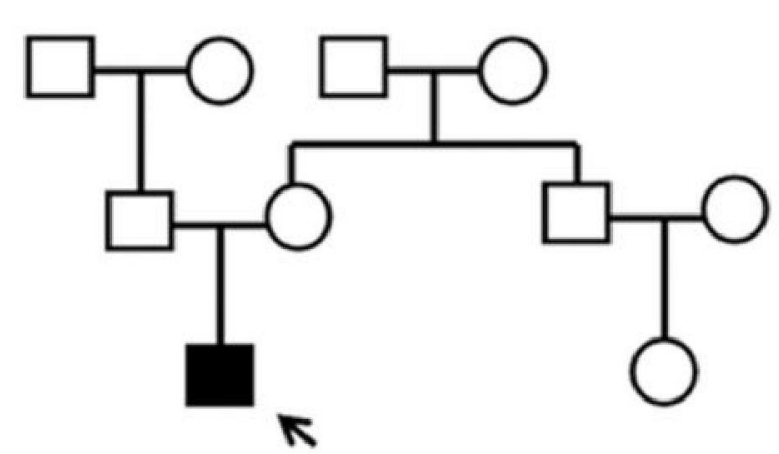

Fig 1 Pedigree of the patient

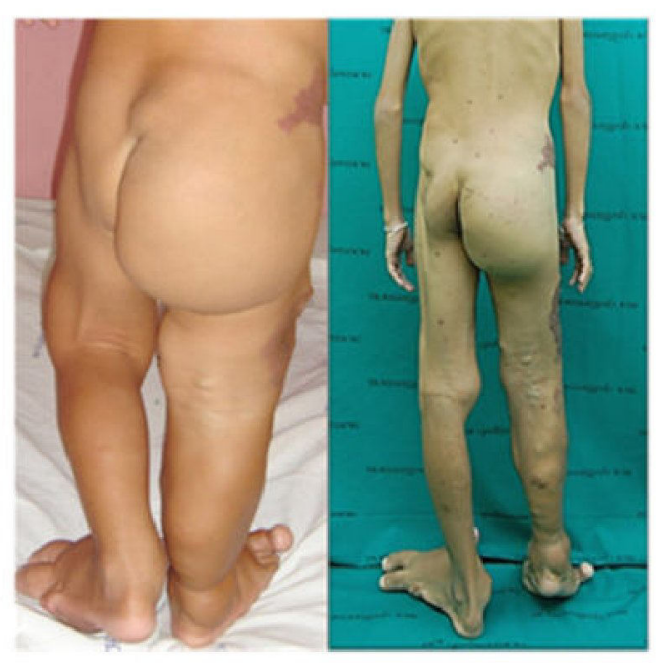

Fig 2 Fig 2 Hemi-hypertrophy of the right buttock and right leg during 3 and 9 years old

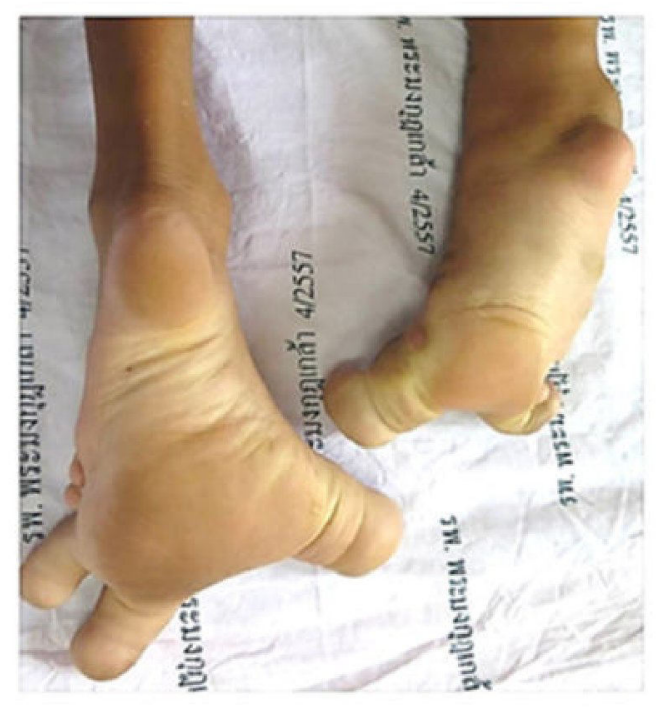

Fig 3 Marked thickening of the soles 


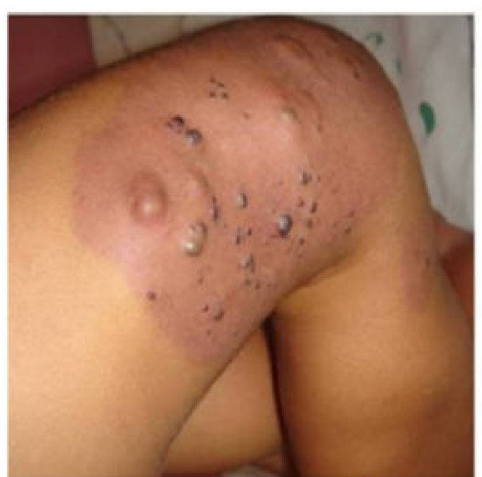

Fig 4 Lymphatic, capillary and venous malformations on the right leg

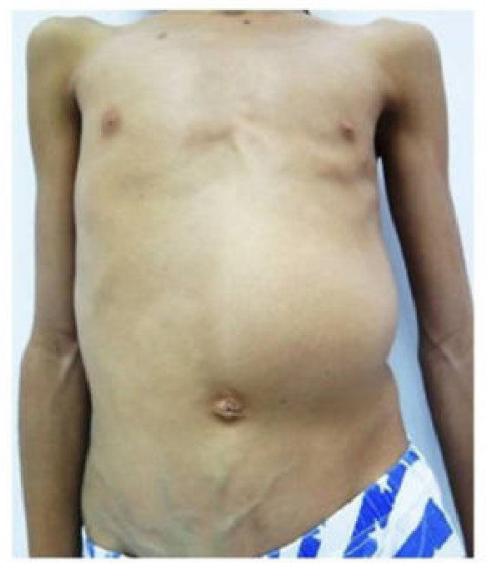

Fig 5 Lipomas with superficial vein dilatation on abdomen

The x-ray of both feet showed enlarging of bones and soft tissue. MRI/MRA of extremities showed disproportionate overgrowth with combined lymphatic-venous malformation at right buttock and right lower extremity.

The venogram of this patient documented patent of deep venous system and incompetent perforating veins at the upper portion of the proximal right leg. Ultrasonography was performed to confirm the segment of venous malformation in which we measured venous diameter and length for the complete preoperative planning.

We treated our patient with endovenous radiofrequency ablation (EV-RFA) by using VNUS $^{\circledR}$ Radiofrequency Generator Model RFG2 (VNUS) Medical Technologies Inc., San Jose, California, USA), and applying the closure with the ablation tip three centimeters in length. He also received the adjunctive treatment with foam sclerotherapy after ablation for the venous malformation of the right leg. Then the limb compression was done with elastic bandage during early postoperative period.
The EV-RFA was performed 3 consecutive times with favorable results. The symptoms and signs including leg pain, feeling of leg heaviness, reducing of the leg size and bleeding problems were very much improved. He also reported the improvement in the abilities for ambulation and in quality of life.

\section{Discussion}

Proteus syndrome is a sporadically occurring hamartomatous disorder. It was first described by Cohen and Hayden in 1979 and designated as Proteus syndrome by Wiedemann et al. $^{(1,2)}$ This condition is characterized by cerebriform connective tissue nevus, epidermal nevi, vascular malformations, lipomas, dermal hypoplasia and hyperpigmentation. Progressive, asymmetric disproportionate overgrowth of body parts are also characteristics of Proteus syndrome. Cerebriform connective tissue nevus is considered as unique pathognomonic sign. ${ }^{(3)}$ The average age of onset for connective tissue nevus to develop is 2 years old. However, during the 6 years follow-up, we did not observe this important sign in our patient. Then our patient is a case of Proteus syndrome without the characteristic cerebriform type connective tissue nevus.

Proteus syndrome is an extremely rare disorder with less than 100 affected individuals reported worldwide. ${ }^{(4)}$ Approximately $90 \%$ of individuals who meet the clinical criteria were caused by an activating somatic mutation of the AKT1 gene (c.49G $>$ A or p. Glu17Lys). ${ }^{(5)}$ AKT is a member of $\mathrm{PI} 3 \mathrm{~K} / \mathrm{AKT} / \mathrm{mTOR}$ signaling pathway which normally plays an essential role in regulation of normal cell growth and survival. Post - zygotic mutation of AKT1 gene results in mosaic distribution and sporadic occurrence of this syndrome. Clinical variability is correlated with the degree of mosaicism of the mutation in the affected cells and tissues.

The mainstay of management in Proteus syndrome needs multidisciplinary approach focusing on disease progression, possible interventions, complication and enhancing the abilities for activities of daily living without difficulty.

Furthermore, the aim of the treatment is to minimize the physical and psychosocial consequences. The individual hamartomatous and localized overgrowth can sometimes be possibly treated surgically. 
Antithrombotic prophylaxis should be considered during surgical procedure because of the potential risks of developing deep venous thromboses and fatal pulmonary emboli. ${ }^{(6)}$ Limb gigantism usuallyrequires vascular imaging and selective embolization. The venogram of ourpatient showed patent of deep venous system and incompetent perforating veins at the upper portion of the proximal right leg. He was a good candidate of receiving treatment with endovenous radiofrequency ablation (EV-RFA) for the venous malformation of his right leg. Then our patient was successfully treated with EV-FRA together with foam sclerotherapy after ablation for the venous malformation of the right leg with favorable outcomes.

\section{Conclusion}

We reported a case of Proteus syndrome that fulfilled the diagnostic criteria which included vascular malformations, lipomas, progressive asymmetric, distorting limb overgrowth and the facial phenotype. (7) Our patient was successfully treated with EV-RFA and foam sclerotherapy for the venous malformation of his leg with promising outcomes. Therefore, endovenous radiofrequency ablation may be an effective option providing alternate or adjunctive treatment modality to surgery for the patients with Proteus syndrome.

\section{References}

1. Cohen MM Jr, Hayden PW. A newly recognized hamartomatous syndrome. Birth Defects Orig Artic Ser 1979;15: 291-6.

2. Wiedemann HR, Burgio GR, Aldenhoff P, Kunze J, Kaufmann HJ, SchirgE. The proteus syndrome. Partial gigantism of the hands and/or feet, nevi, hemihypertrophy, subcutaneous tumors, macrocephaly or other skull anomalies and possible accelerated growth and visceral affections. Eur J Pediatr 1983; 140: 5-12.

3. Cohen MM Jr. Proteus syndrome review: molecular, clinical, and pathologic features. Clin Genet 2014; 85 : 111-9.

4. Cohen MM Jr. Proteus syndrome: an update. Am J Med Genet C Semin Med Genet 2005; 137: 38-52.

5. Lindhurst MJ, Sapp JC, Teer JK, Johnston JJ, Finn EM, Peters K, et al. A mosaic activating mutation in AKT1 associated with the Proteus syndrome. N Eng J Med 2011;365: 611-9.

6. Cohen MM Jr. Causes of premature death in Proteus syndrome. Am J Med Genet 2001; 101: 1-3.

7. Turner JT, Cohen MM Jr, Biesecker LG. Reassessment of the Proteus syndrome literature: application of diagnostic criteria to published cases. Am J Med Genet A 2004; 130: 111-22. 